\title{
Comparing the Effect of Risperidone, Virtual Reality and Risperidone on Social Skills, and Behavioral Problems in Children with Autism: A Follow-up Randomized Clinical Trial
}

\author{
Sakineh Soltani Kouhbanani, $\mathrm{PhD}^{1 *}$; Razieh Khosrorad, PhD²; Somayeh Zarenezhad, ${ }^{2}{ }^{1}$; Seyedeh Manizheh Arabi, PhD ${ }^{3}$ \\ ${ }^{1}$ Department of Educational Sciences, Educational Sciences and Psychology Faculty, Ferdowsi University of Mashhad, Mashhad, \\ Iran \\ ${ }^{2}$ Department of Health Education, Health Faculty, Sabzevar University of Medical Sciences, Sabzevar, Iran \\ ${ }^{3}$ Department of Motor Behavior, Faculty of Physical Education and Sport Sciences, Bu-Ali Sina University, Hamedan, Iran
}

\begin{abstract}
Background: Improving behavioral and social problems in children with autism requires extensive training programs even with parents at home. The main goal of this study is to design a virtual reality (VR) intervention based on the TEACCH method in combination with risperidone to evaluate its effectiveness on social and behavioral problems.

Methods: Forty-three children with autism (6-12 years old) randomly were divided into three groups: risperidone $(n=15)$, risperidone + VR $(n=15)$, and control $(n=13)$. The interventions lasted for 3 months (90 sessions) and post-test assessments were done immediately after intervention. Follow up tests were done 3 months after that.

Results: Risperidone + VR group showed significant differences in social skills (MD=36.59; 95\% Cl: 30.74 to $38.42, \mathrm{P}<$ $0.001, \mathrm{y} 2=1.51$ in post-test; $\mathrm{MD}=19.63 ; 95 \% \mathrm{Cl}: 17.27$ to $21.63, \mathrm{P}<0.001, \mathrm{y} 2=0.86$ in follow up); and behavioral symptoms $(\mathrm{MD}=-36.12 ; 95 \% \mathrm{Cl}:-39.72$ to $-36.91, \mathrm{P}<0.001, \mathrm{n} 2=1.99$ in post-test; $\mathrm{MD}=-28.82 ; 95 \% \mathrm{Cl}:-29.43$ to $-25.32, \mathrm{P}<0.001$, $\mathrm{n} 2=1.58$ in follow up) compared to the control group. However, the risperidone group showed significant differences in social skills ( $\mathrm{MD}=2.03 ; 95 \% \mathrm{Cl}$ : 0.82 to $3.67, \mathrm{P}<0.001, \mathrm{y} 2=0.12$ ) and behavioral symptoms ( $\mathrm{MD}=-36.66 ; 95 \% \mathrm{Cl}:-38.96$ to -34.27 , $\mathrm{P}<0.001, \mathrm{y} 2=1.96)$ only in post-test. Thus, the experimental groups did not have any significant difference in post-test.

Conclusion: Combined interventions such as VR can enhance the effectiveness of risperidone response and boost children's preparedness to practice and learn social interaction.

Keywords: Autism spectrum disorder, Behavioral problems, Risperidone, Social skills, Virtual reality

Cite this article as: Soltani Kouhbanani S, Khosrorad R, Zarenezhad S, Arabi SM. Comparing the effect of risperidone, virtual reality and risperidone on social skills, and behavioral problems in children with autism: a follow-up randomized clinical trial. Arch Iran Med. 2021;24(6):534-541. doi: 10.34172/aim.2021.76
\end{abstract}

Received: July 8, 2020, Accepted: September 13, 2020, ePublished: July 1, 2021

\section{Introduction}

The most obvious characteristics of children with the autism spectrum disorder (ASD) are difficulty in social communication and behavioral difficulties, representing its most enduring features that may even continue into adulthood. ${ }^{1}$ Such behaviors give rise to a myriad of developmental difficulties in children with ASD and they serve as a source of stress for their parents. ${ }^{2}$ One of the most common therapeutic modalities to diminish behavioral difficulties in children with ASD is risperidone. It has been shown to improve hyperactivity, speech disorders, social isolation, and repetitive behaviors in some children with ASD. ${ }^{3,4}$ However, after the discontinuation of risperidone, the behavioral disorders relapse and return to their original level. ${ }^{5}$ This indicates the need for long-term use of drugs to diminish behavioral difficulties. Although there are individual differences regarding the side effects of taking this medication, the reported side effects of risperidone outweigh its benefits. Weight gain and metabolic disorder are common side effects reported in these children. In a study, the effect of 24-week risperidone treatment versus parental training plus risperidone was investigated in 124 children with ASD with behavioral problems. During the experiment, body mass index, waist circumference and side effects were controlled. According to the results of this study, the group taking risperidone demonstrated significant weight gain compared to the other group. Syndrome of metabolic and insulin resistance may occur as a consequence of fast weight gain. The growing use of this medication in children with ASD, ${ }^{6}$ the ineffectiveness of this medical intervention in all children with ASD, 3,4 as well as the extreme side effects of this medication and its deleterious effects on physical health have encouraged combined interventions (medication with behavioral interventions) to reduce the dose of this drug in a bid to allay the sufferings of autistic children. Hence, considering these factors, we designed an intervention to examine whether using virtual reality (VR) in combination with 
risperidone could alleviate maladaptive behaviors, including social isolation and behavioral difficulties in children with ASD.

Since the dawn of the new millennium, there has been an explosion of interest in technology, especially in the field of VR education and it has been extensively utilized to address the problems of certain demographic groups such as people with phobias or disabilities. ${ }^{7}$ Today, applied research studies in VR provide greater opportunities for developing autism rehabilitation programs. VR creates a platform for children with ASD to participate in a range of activities that can reduce their social and behavioral problems. VR can be a means of generating cognitive and behavioral experiences for children with ASD before they enter the real world and encounter these stimuli. ${ }^{8}$ Previous studies on VR applications have reported enhanced detection of other people's feelings by children with ASD (theory of mind), social functioning, and mitigated behavioral problems in children with ASD. ${ }^{9}$ Most VR interventions involve a number of visual experiences displayed on the monitor screen or in a room designated for such a display. Ip et aldesigned a room where children with ASD could practice social interactions in different environments using VR. The results of this study suggested that their social functioning and emotion control improved significantly after 24 weeks of VR exercise. ${ }^{10}$ One case study based on VR demonstrated that VR was only successful in allaying phobia in certain children with ASD, and this type of intervention was welcomed by only some of them. ${ }^{11}$ This study also involved a room where cognitive exercises were practiced through VR. It seems that bringing children into such a room stirred up a huge emotional load, which may explain why children with ASD reject this type of intervention. ${ }^{12}$ In this type of intervention, the child needs to be present at the place of intervention, and they will not be as effective in environments familiar to the child or when interventions are implemented by parents. Given the ASD children's fascination with technology and media, and the diminished emotional burden imposed on the child with ASD by unfamiliar environments, we determined to design the intervention program based on VR so that children could practice these skills at home where they spend most of their time using the most basic three-dimensional instruments. On the other hand, the program can be run by parents at home, and the execution process could be followed up by a child therapist.

For autistic children, practicing at home offers several benefits. One thing that contributes to the effectiveness of behavioral exercises is dedicating a great amount of time to these exercises. Children with ASD need to be subjected to extensive behavioral treatments, part of which should be conducted at home. Moreover, since children spend a great amount of their time at home, parents can undertake a share of the treatment at home. However, it seems that the process of behavioral therapies at home needs to be monitored by a psychologist to give parents necessary guidance and the accuracy of exercises have to be supervised by experts. These factors necessitate the design of a parent-centered treatment plan that could be monitored by psychologists. One of such parent-centered behavioral therapy programs for children with ASD is the TEACCH program. It covers a series of exercises formatted to manage age-appropriate skills. ${ }^{13}$

Our first hypothesis is that TEACCH intervention by VR coupled with risperidone can contribute to the reduction of behavioral and social difficulties. The second hypothesis of this study is to examine the durability of benefits derived from VR intervention combined with risperidone versus the effects of risperidone alone. Accordingly, the major purpose of the current paper is to investigate the effect of VR program based on TEACCH intervention on behavioral difficulties and social skills, in children with ASD aged 6-12 years.

\section{Materials and Methods \\ Trial Design}

In this study, a pretest-posttest design with control group and three months follow-up assessment was adopted for 6 to 12 year-old children with ASD. In this study, there were three groups (i.e. risperidone, VR + risperidone, or a passive control group) with a 1:1:1: ratio, where the participants were randomly allocated to each group. This study was conducted in behavior and mental health clinic in Mashhad, Khorasan Razavi province, Iran, from February 2018 to July 2019. The study was conducted in compliance with principle of the Declaration of Helsinki. All children's parents signed the consent form. In addition, they were told that they could quit the study at any time they wished. Parents and their children referred to the clinic and pre-test evaluations were performed two weeks in advance of the intervention. The interventions lasted for 3 months (90 sessions) and post-test assessments were done immediately after the intervention. Follow-up tests were done 3 months after that.

\section{Participants}

The participants were randomly selected from among 102 ASD children referred to the Mental and Behavioral Center in 2018-2019. Their parents also took part in the study. Participants attended the clinic for screening, baseline assessment, after the intervention and three months later. The inclusion criteria for selecting the participants were age between 6 and 12 years at the time of enrollment, and suffering from ASD, which was confirmed by a neurologist and psychologist. The confirmation was achieved base on the result of the Diagnostic and Statistical Manual of Mental Disorders (DSM-5) and Autism Diagnosis Interview-Revised (ADI-R). The other criterion was a non-verbal cognitive score at the level of $>70$ (children's cooperative ability determines this IQ cutoff score). The exclusion criteria were suffering from attention deficit/ hyperactivity disorder, seizure and fragile $\mathrm{X}$, visual, auditory or motor impairment, and absence of 
both parents at the time of intervention. All subjects had a primary diagnosis of autism endorsed by a psychiatrist and their autism was further confirmed by the Childhood Autism Rating Scale (CARS). All participants had low to moderate IQ based on the Wechsler Intelligence Scale (See Table 1).

\section{Virtual Reality + Risperidone Experimental Group}

The intervention program was planned by a specialist and parents took over its implementation and followed up at home. The management software was entirely designed on the specialist's smartphone (Phone 1), which consisted of individualized programs tailored to the needs of each child. Phone 1 acts as the brain system sending 3D images and videos to Phone 2. Applications were sent to Phone 2 used by parents, and they used the Bluetooth connection to transfer the intervention to the VR glasses worn by the children.

Parents undertook this intervention at home, and the confirmation and completion of each step of the intervention was saved and sent by parents from Phone 2 to Phone 1. Programs are generally designed to (1) help parents work out how autism disorder can influence children's behavior; (2) how they can aid their child to perform daily activities conveniently, and (3) identify growth programs that fit their children so that they can interact and play with them in a more effective way. In this program, one parent is assumed to be in charge of intervention and sending of information for each child. For 90 sessions, the children were subjected to $45-\mathrm{min}$ sessions of VR TEACCH on a daily basis (seven days per week).

After the intervention, a post-test was performed akin to the pre-test and a follow-up test was run to evaluate the viability of intervention after 3 months. The intervention program was designed by an exceptional children's specialist who had experience working with autistic children. Initially, a meeting was held in which the parents of each child and the therapist were present. In this session, the parents shared their child's information with the therapist, stated their intervention priorities, and received the TEACCH intervention program to start intervention at home. Also, they learned how to use the apps on the phone. The intervention comprised activities such as recognizing colors and names of geometric shapes, self-help activities such as washing hands and brushing, and fine motor skills such as tying shoelaces and wearing clothes. ${ }^{14}$ The purpose of these interventions was to stress cognitive and spatial, processing and visual memory, so that the children could easily perform their daily activities. ${ }^{15}$

The risperidone dose was prescribed by a pediatric psychiatrist who was blind to the study. Generally, risperidone dose is prescribed based on the child's weight. Children weighing between 14 and $20 \mathrm{~kg}$ are given 0.25 $\mathrm{mg} / \mathrm{d}$ of risperidone, which increases progressively to 1.75 $\mathrm{mg} / \mathrm{d}$. For older patients who weigh 20 to $40 \mathrm{~kg}$, the dose starts at $0.5 \mathrm{mg}$ daily and gradually reaches a maximum of $2.25 \mathrm{mg}$ daily. For children over $45 \mathrm{~kg}$, the dose starts at $0.5 \mathrm{mg} / \mathrm{d}$ and increases steadily to $3.5 \mathrm{mg} / \mathrm{d}$. During the intervention, if the side effects of taking risperidone such as weight gain exceeded normal levels, the specialist was allowed to postpone the dose increase or change the type of drug used, which did not happen for our participants.

\section{Risperidone Experimental Group}

Another intervention group was also present in the study, which only received drug treatment. The risperidone dose in this group was also determined by the psychiatrist in the same way as for the other experimental group. During the intervention, none of the participants in the two experimental groups showed any adverse effects associated with risperidone use, and their dose of risperidone was increased according to the schedule relative to their weight.

\section{Control Group}

During the intervention, the control group did not have any medication or behavioral intervention, but if the child's behavioral problems were intensified, the psychiatrist had permission to prescribe risperidone therapy for these children. In this case, the participant would be removed from the intervention. During the intervention period, two participants had to take medication and were therefore withdrawn from the study. At the termination of the investigation, to comply with the ethical standards, the control group received drug therapy along with VR intervention and the group only using drug received VR

Table 1. Descriptive Statistics for Chronological Age, IQ, CARS II Scores in Each Groups

\begin{tabular}{|c|c|c|c|c|}
\hline & & Risperidone $(n=15)$ & Risperidone + VR $(n=15)$ & Control $(n=15)$ \\
\hline \multicolumn{2}{|c|}{ Chronological age } & $8.44(1.94) ; 6-11^{\mathrm{a}}$ & $8.59(2.12) ; 6-12$ & $8.40(2.01) ; 6-12$ \\
\hline \multicolumn{2}{|c|}{ IQ } & $71.22(8.20) ; 6-82$ & 72.37 (8.20); 60-85 & 78.20 (8.44); 60-85 \\
\hline \multicolumn{2}{|c|}{ CARS } & 95.78 (19.76); 70-120 & 91.25 (22.65); 64-125 & $95.50(20.10) ; 73-124$ \\
\hline \multirow{2}{*}{ Gender } & Male & $73 \%$ & $75 \%$ & $69 \%$ \\
\hline & Female & $27 \%$ & $25 \%$ & $31 \%$ \\
\hline \multicolumn{2}{|c|}{ Maternal education (y) } & $14.23(2.45)$ & $13.67(1.39)$ & $14.43(2.14)$ \\
\hline \multicolumn{2}{|c|}{ Paternal education (y) } & $13.65(5.21)$ & $13.38(1.25)$ & $14.87(3.64)$ \\
\hline
\end{tabular}

IQ, Intelligence Quotient; CARS, Childhood Autism Rating Scale; VR, Virtual reality.

${ }^{a}$ Data presented as mean $(\mathrm{SD})$; range. 
intervention for three months.

\section{The Children Autism Rating Scale II Test}

The CARS-II, which is based on the definition of American Autism Society and the American Psychiatric Association, relies on the DSM-V to help diagnose autism in people aged 3 to 22 years. The CARS- 2 test consists of 42 items in three subscales (stereotyped behavior, communication and social interaction) that are completed in the study. In addition, the scale contains 14 items about developmental disabilities, which are not considered as part of the pre-test and post-test scores. On this scale, raw scores are transmuted to autism index scores, and scores higher than 90 with a probability greater than mean and scores less than 90 with a probability lower than mean confirm the diagnosis of autism. In fact, the higher scores in this scale demonstrate the greater severity of the disorder and lower scores denote a mild disorder. CARS's reliability is indicated by an alpha coefficient of 0.89 for stereotypical behaviors, 0.74 for communications, 0.92 for social interactions, 0.73 for developmental disorders, and 0.80 for autism semiotics. The validity of the test has been evaluated through comparison with other autism diagnostic tools. ${ }^{16}$

\section{Vineland Adaptive Behavior Scale}

This 297-item scale measures a child's adaptive behaviors from birth to the age of 18 and 11 months. The items are scored in the range of 0 to 2 based on the criteria assigned to each item. In general, items that are performed habitually receive 2 points; items that are done occasionally or the subject is partially able to perform receive 1 point, and items that are rarely or never conducted receive a zero point. The test-retest reliability coefficient of the whole test has been estimated in the range of 0.80 to 0.90 . This value is 0.78 to 0.94 for the socialization domain and 0.70 to 0.95 for the motor domain. ${ }^{17}$

\section{Sample Size and Power}

Calculations to establish the sample size were performed using power analysis and using an effect size from pervious study that tested the effect of VR training on social adaption of children with ASD. ${ }^{10}$ The significance level was set at $\alpha=0.05$. According to the standard deviation established for social adaption in the previous study and power of 0.85 , a valid sample size was calculated at $n=36$ (each group contained 12 participants). Anticipating three withdrawals in each group, the sample size was determined at 15 participants in each group: $V R+$ risperidone $(n=15)$, risperidone $(n=15)$ and control group $(n=15)$.

\section{Randomization, Allocation and Administration}

The eligible participants were randomly assigned to either the experimental or control groups using random transpose blocks (1:1:1: ratio) by the first author. She also enrolled the participants and assigned them to each study group. Two weeks before the allocation, the cognitive level and autism severity of 102 children with ASD referred to the Mental and Behavioral Center in 2018-2019 were assessed. Among them, 45 participants who had eligible criteria were chosen to participate in the study. Participants included 30 boys and 15 girls with ASD with 2:1 ratio. While parents of children and the first author were aware of the allocated arm, outcome assessors and data analysts were preserved blind to the allocation. One week before the intervention, the pre-test was performed for all participants. After 90 sessions of training, 15 participants in the $\mathrm{VR}+$ risperidone group, 15 participants in the risperidone group and 13 participants in the control group were assessed again for posttest. Two participants in control group had to take medication and were therefore withdrawn from the study. Also, the followup test was run to evaluate the viability of intervention after 3 months. Overall, 43 participants completed the intervention ( $n=13$ in the control group, and $\mathrm{n}=15$ in each of the VR + risperidone and risperidone groups); the power of the test still was high at 0.85 . Figure 1 illustrates the recruitment, enrollment, randomization and followup on a CONSORT diagram.

\section{Statistical Methods}

Group means (M) and standard deviations (SD) were used in this study. A two-way repeated measure analysis of co-variance was administrated to determine the effect of interventions and measurements across time on social skills and behavioral symptoms. The dependent variables were Villand social skill and CARS behavioral symptoms scores. The within-individual factors were intervention groups (risperidone, risperidone $+\mathrm{VR}$, control) and time (pre-test, post-test, 3-month follow up). The main effect of time and groups, as well as the group $\times$ time interaction effect were analyzed. The alpha level of 0.05 was considered for finding significant differences between the means across time. The eta-square statistic (n2) was computed to estimate the effect size to distinguish the practical significance of findings. Typical assumptions of analysis of variance (ANOVA), such as normality and homogeneity of variance, were checked. $\mathrm{R}$ statistical software (version 3.4.3) was used for analyzing the data.

\section{Results}

\section{Comparison of Social Skill}

The results of one-way analysis of variance showed that there were no significant differences between the experimental and control groups in the pre-test mean scores of social skills $\left(\mathrm{F}_{(2,40)}=0.31, P=0.754\right)$.

To test the underlying assumptions, analysis of covariance with repeated measures design was used. After controlling for variables including age, intelligence, and autism severity, the repeated measures analysis of covariance (ANCOVA) indicated that at the 0.05 level, the model was significant. The results reveal the significant main effects of group $\left(F_{(2,40)}=528.944, P<0.001\right.$, $\mathrm{\eta} 2=0.46)$, time $\left(\mathrm{F}_{(2,40)}=658.505, P<0.001, \mathrm{n}^{2}=0.41\right)$, and 


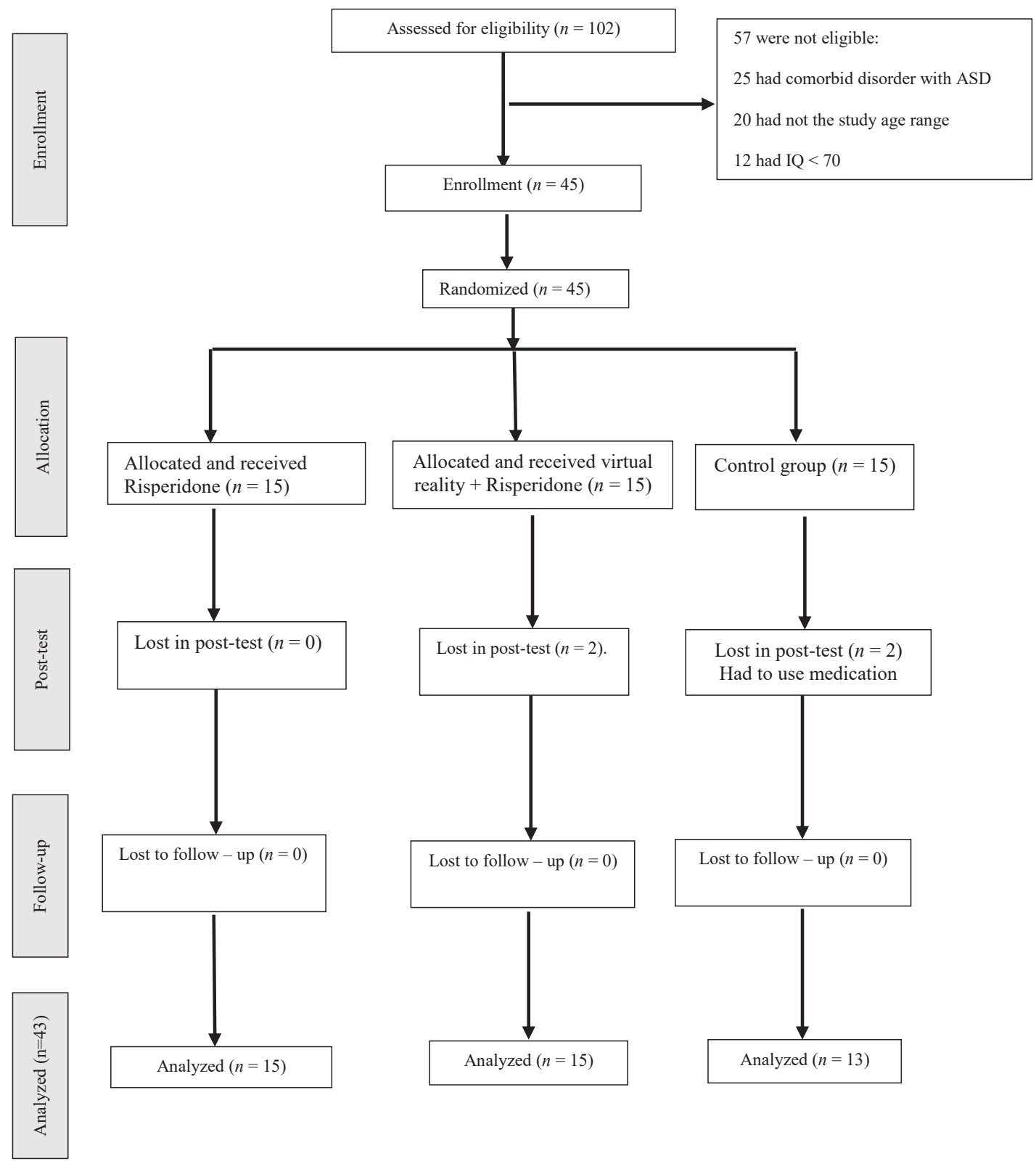

Figure 1. Participant Recruitment, Enrollment, Randomization and Follow-up Through Each Stage of the Study.

a significant interaction between group and time $\left(\mathrm{F}_{(4,}\right.$ 40) $=197.236, P<0.001, \mathrm{n}^{2}=0.42$ ) for social skills.

Pairwise comparison using paired t-test with a Bonferroni adjustment showed significant mean differences in social skill scores between pre-test and posttest $(\mathrm{MD}=37.73$; 95\% CI: 18.31 to $38.43, P<0.001)$ and between pre-test and 3-month follow-up $(\mathrm{MD}=27.264$; 95\% CI:14.52 to $31.43, P<0.001)$ in the risperidone + VR group. In addition, significant mean differences in social skill scores were observed between pre-test and post-test $(\mathrm{MD}=29.866$; 95\% CI: 20.51 to $31.22, P<0.001)$ and non-significant mean differences between pre-test and 3-month follow-up $(\mathrm{MD}=4.93$; 95\% CI: 1.32 to 5.61, $P=0.843)$ in the risperidone group. The results of comparison between groups indicated that in posttest, risperidone + VR showed a significant increase in social skill scores in comparison with the control group $\left(\mathrm{MD}=36.59\right.$; $95 \% \mathrm{CI}: 30.74$ to $\left.38.42, P<0.001, \mathrm{n}^{2}=1.51\right)$.
Also, the risperidone group showed a significant increase in social skill score in comparison with the control group $\left(\mathrm{MD}=2.03 ; 95 \% \mathrm{CI}: 0.82\right.$ to $\left.3.67, P<0.001, \mathrm{n}^{2}=0.12\right)$, while there was no significant difference between the risperidone and risperidone $+\mathrm{VR}$ groups in social skill scores $(\mathrm{MD}=36.56 ; 95 \% \mathrm{CI}: 31.52$ to $37.07, P=0.643$, $\mathrm{n}^{2}=1.34$ ). In the follow-up, only the risperidone $+\mathrm{VR}$ group revealed a significant difference in comparison with the control group $(\mathrm{MD}=19.63 ; 95 \% \mathrm{CI}$ : 17.27 to 21.63, $P<0.001, \mathrm{n}^{2}=0.86$ ), while there was no significant difference between the risperidone and control groups $\left(\mathrm{MD}=-1.37 ; 95 \% \mathrm{CI}:-2.76\right.$ to $\left.0.94, P=0.751, \mathrm{n}^{2}=0.07\right)$. The results are illustrated in Figure 2.

\section{Comparison of Behavioral Symptoms}

The results of one-way analysis of variance showed that there were no significant differences between the experimental and control groups in the pre-test mean 


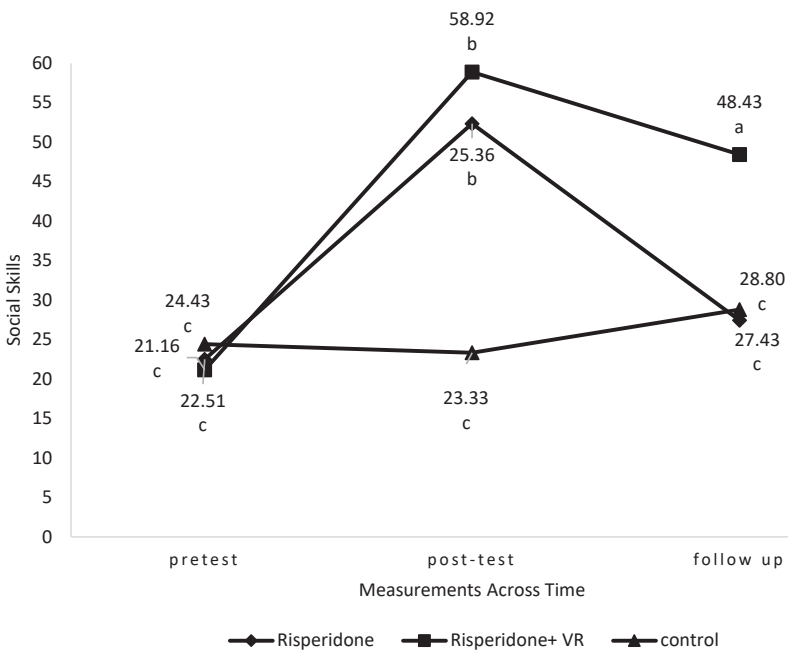

Figure 2. Scores of Experimental and Control Groups in Social Skills in Pre-test, Post-test and Follow-up. Same letter for each two by two comparison means that there is not any significantly differences.

scores of behavioral symptoms $\left(\mathrm{F}_{(2,40)}=0.43, P=0.627\right)$.

The repeated measures ANCOVA showed that with controlling for variables such as age, intelligence, and autism severity, the model is significantly fit at the 0.05 level. The results reveal the significant main effects of group $\left(\mathrm{F}_{(2,40)}=26.482, P<0.001, \mathrm{n} 2=0.37\right)$, time $\left(\mathrm{F}_{(2,40)}=20.531\right.$, $\left.P<0.001, \mathrm{n}^{2}=0.20\right)$, and a significant interaction between group and time $\left(\mathrm{F}_{(4,40)}=6.784, P<0.001, \mathrm{n}^{2}=0.29\right)$ for behavioral symptoms.

Pairwise comparison using paired t-test with a Bonferroni adjustment indicated significant mean differences in behavioral symptom scores between pretest and post-test $(\mathrm{MD}=-28.41 ; 95 \% \mathrm{CI}:-12.98$ to -40.23 , $P<0.001)$ and between pre-test and 3-month follow-up $(\mathrm{MD}=-7.067 ; 95 \% \mathrm{CI}:-1.67$ to $-8.39, P<0.001)$ in the risperidone + VR group. Also, significant mean differences in behavioral symptom scores were found between pretest and post-test $(\mathrm{MD}=-28.53$; $95 \%$ CI: -15.68 to -29.92 , $P<0.001)$ and non-significant differences between pretest and 3-month follow-up ( $\mathrm{MD}=-10.734 ; 95 \% \mathrm{CI}$ : -1.79 to $-11.67, P=0.627)$ in the risperidone group. The results of comparison between groups indicated that in post-test, risperidone + VR showed a significant decrease in behavioral symptom scores in comparison with the control group ( $\mathrm{MD}=-36.12$; 95\% CI: -39.72 to -36.91 , $\left.P<0.001, \mathrm{n}^{2}=1.99\right)$. Also, the risperidone group showed a significant decrease in behavioral symptom scores in comparison with the control group ( $\mathrm{MD}=-36.66$; $95 \%$ CI: -38.96 to $-34.27, P<0.001, \mathrm{n}^{2}=1.96$ ), while behavioral symptoms scores did not have any significant difference between the risperidone and risperidone + VR groups $\left(\mathrm{MD}=1.27 ; 95 \% \mathrm{CI}: 0.82\right.$ to $\left.2.05, P=0.075, \mathrm{n}^{2}=0.01\right)$. In the follow-up, only the risperidone $+\mathrm{VR}$ group revealed a significant difference in comparison with the control group ( $\mathrm{MD}=-28.82$; 95\% CI: -29.43 to -25.32 , $P<0.001, \mathrm{n}^{2}=1.58$ ), while the risperidone and control groups did not have any significant difference with each other $(\mathrm{MD}=-16.16$; $95 \% \mathrm{CI}:-19.79$ to $-15.37, P=0.213$, $\left.\mathrm{n}^{2}=0.72\right)$. The results are presented in Figure 3 .

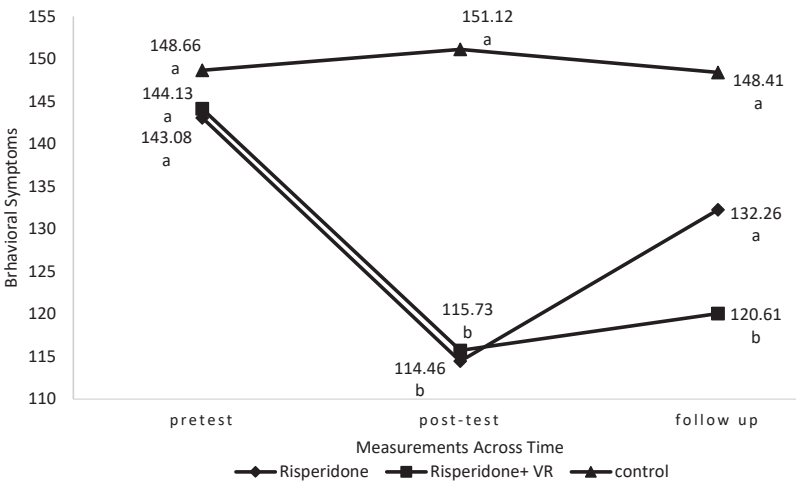

Figure 3. Scores of Experimental and Control Groups in Behavioral Symptoms in Pre-test, Post-test and Follow-up. Same letter for each two by two comparison means that there is not any significantly differences.

\section{Discussion}

Given the importance of social interactions and communication skills in one's life, relationships, and educational process, and considering the serious problems of children with ASD, the purpose of this investigation was to evaluate the effectiveness of an educational program designed for children with ASD using VR and risperidone therapy on behavioral and social problems. In general, the results of the post-test demonstrated a significant improvement in behavioral problems and social skills of both experimental groups. Follow-up test outcomes showed that three months after the end of interventions, the social and behavioral problems of the group receiving only risperidone relapsed to their original state, while in the VR + risperidone group, the relapse of behavioral disorders was not as significant and they exhibited a substantial improvement in behavioral and social problems compared to the group receiving only risperidone. This study revealed that medication therapy alone cannot significantly alleviate the social and behavioral problems in ASD children when the medication is not used, while VR training improved social and behavioral problems. Therefore, it might be expected that VR interventions exert a greater impact on these problems. The findings of this study are well aligned with those of Aman et al. In their study, a combination of risperidone and behavioral intervention had a significant effect on mitigation of behavioral problems compared to medication alone. ${ }^{18}$ Various studies have documented that risperidone has a meaningful effect on reducing stereotypic behavior ${ }^{19}$ and it seems that similar results observed in this study regarding the mitigation of stereotyped behaviors in both intervention groups are induced by risperidone. The effect of this medication on stereotyped behaviors does not come as a surprise as previous studies have reported the comparable effect of haloperidol for improving stereotyped behaviors. ${ }^{20}$ In a study by McDougle et al, the therapeutic effect of risperidone was evaluated on adults and children with ASD. The results revealed that risperidone had a positive effect on some children's behavioral symptoms and pervasive developmental 
disorders and could mitigate their behavioral disorders. ${ }^{19}$ In 2002, McCracken et al investigated the effect of risperidone for treatment of children with ASD aged 5 to 17 years. In their research, 49 subjects were treated with risperidone and 52 with placebo for 8 weeks. The findings suggested reduced restlessness in $52.9 \%$ of participants in the experimental group compared to $14.1 \%$ in the placebo group. ${ }^{21}$ In a review article by Canitano and Skandora regarding the treatment of autism with risperidone, the authors found that risperidone moderates positive effects on communication and social difficulties. ${ }^{22}$

In keeping with the results of the present study, other studies have demonstrated that risperidone alone does not have a significant effect on social skills; however, they suggest that risperidone, by mitigating a number of stereotyped behaviors, can probably prepare an autistic child to participate in social interactions and improve social isolation. ${ }^{23}$ Our study also reported lower recurrence of social disorders in the group receiving combination therapy or medication at the end of treatment. On the other hand, following the discontinuation of treatment, there was a significant difference between the two groups of medication treatment and combined intervention in terms of social and behavioral disorders. Thus, according to other studies, despite the efficacy of risperidone in treating some behavioral disorders, it has no significant effect on alleviation of major problems of children with ASD and its discontinuation would lead to the recurrence of symptoms. Also, adopting a proper combined program may help to improve these problems and related behavioral disorders and partially diminish the rate of recurrence. The results reported by Beckle et al, Lahiri et al, Bayan et al, and Didehbani et al consistent with the findings of the present study, exhibit the positive impact of VR education on mitigating problems in autistic children. Didehbani et al developed social cognitive educational programs for autistic children with high performance. This study tested the efficacy of social cognitive education based on VR on improving social communication in children with ASD. The participants of this study consisted of 30 ASD children aged 7 to 16 years who took part in the treatment and educational sessions. Virtual environments used in the study covered school classroom, school cafeteria, playground, campground, and so on. Each social scenario was designed with an emphasis on learning specific social items such as meeting new people, joining a group of friends, and consulting with a friend. The results revealed improvement in measures of emotional cognition, social authority, executive performance and deductive reasoning. ${ }^{24}$ The harmful consequences of social disorders may include poor educational progress, social failures, anxiety, depression, abuse, barriers to social communication, and social isolation. ${ }^{25}$ Socializing and the ability to interact with other people is of paramount importance in life, but autistic children are seriously flawed in this regard. For this reason, these children are in need of support and training in social skills to obtain the essential social proficiencies. The findings of this study revealed that the administration of VR is an impressive treatment strategy to improve social deficits in autism.

In general, it can be contended that risperidone mitigates behavioral disorders, preparing children for participation in social interactions. On the other hand, combined interventions can augment the efficacy of this medication and increase the child's preparedness for acquiring social skills. Thus, the synthesis of educational and pharmacological interventions can contribute to rapid and effective resolution of social problems and subsequently, improve communication and social skills in children. In conclusion, VR, as a hybrid approach, offers a new technology to build an appropriate environment for children with ASD to interact with the environment and develop a sense of control. Since the system is virtual, the child is not scared of criticism by the therapist or other authority figures and tries to interact with others. Autistic children are reluctant to see a psychologist or psychiatrist and refuse to carry out exercises or play games. However, when placed in an environment similar to a playground, they will no longer be afraid of adults and attempt to engage in communications with others. Future studies are needed to explore the possibility of using concepts other than real-world realities such as imaginary animals (for example: Angry Birds or Sponge Bob or any other favorite animals or dolls) in a VR environment, and its extension to other characters such as dolls, among other things. This study had a number of limitations including small sample size and short follow-up period. It is recommended for future studies to use larger sample sizes to corroborate the accuracy of the results. Moreover, more longitudinal studies should be conducted to evaluate the effect of treatment. It seems that there is still a significant gap in the treatment of autism, which can be bridged through further research and emphasis on results for better treatment of these patients.

\section{Authors' Contribution}

SSK and SZ are involved in the conception and study design. SMA did the statistical analysis and write the initial version of the study. SSK and RK were project leaders and gathering data. The final manuscript was approved by all authors.

\section{Conflict of Interest Disclosures}

The authors declare that they have no conflict of interest.

\section{Ethical Statement}

Ferdowsi University of Mashhad's Institutional Review Board and Ethics Committee of Bioethics (Registration code: IR.MUM. FUM.REC.1398.129) and the Ethics Committee Board of the Iranian Registry of Clinical Trials (IRCT) have approved this study (identifier: IRCT20191113045429N1).

\section{References}

1. Ozturk Y, Bizzego A, Esposito G, Furlanello C, Venuti P. Physiological and self-report responses of parents of children with autism spectrum disorder to children crying. Res Dev Disabil. 2018;73:31-9. doi: 10.1016/j. 
ridd.2017.12.004

2. Tsai HW, Cebula K, Liang SH, Fletcher-Watson S. Siblings' experiences of growing up with children with autism in Taiwan and the United Kingdom. Res Dev Disabil. 2018;83:206-16. doi: 10.1016/j.ridd.2018.09.001.

3. King BH, Wright DM, Handen BL, Sikich L, Zimmerman AW, McMahon W, et al. Double-blind, placebo-controlled study of amantadine hydrochloride in the treatment of children with autistic disorder. J Am Acad Child Adolesc Psychiatry. 2001;40(6):658-65. doi: 10.1097/00004583200106000-00010.

4. Woodard C, Groden J, Goodwin M, Bodfish J. A placebo double-blind pilot study of dextromethorphan for problematic behaviors in children with autism. Autism. 2007;11(1):29-41. doi: 10.1177/1362361307070989.

5. Scheiman M, Mitchell GL, Cotter S, Cooper J, Kulp M, Rouse $\mathrm{M}$, et al. A randomized clinical trial of treatments for convergence insufficiency in children. Arch Ophthalmol. 2005;123(1):14-24. doi: 10.1001/archopht.123.1.14.

6. Levine SZ, Kodesh A, Goldberg Y, Reichenberg A, Furukawa TA, Kolevzon A, et al. Initial severity and efficacy of risperidone in autism: Results from the RUPP trial. Eur Psychiatry. 2016;32:16-20. doi: 10.1016/j. eurpsy.2015.11.004.

7. Fitzgerald E, Yap HK, Ashton C, Moore DW, Furlonger $\mathrm{B}$, Anderson A, et al. Comparing the effectiveness of virtual reality and video modelling as an intervention strategy for individuals with autism spectrum disorder: brief report. Dev Neurorehabil. 2018;21(3):197-201. doi: 10.1080/17518423.2018.1432713.

8. Lorenzo G, Lledó A, Pomares J, Roig R. Design and application of an immersive virtual reality system to enhance emotional skills for children with autism spectrum disorders. Comput Educ. 2016;98:192-205. doi: 10.1016/j. compedu.2016.03.018.

9. Yang YJD, Allen T, Abdullahi SM, Pelphrey KA, Volkmar FR, Chapman SB. Neural mechanisms of behavioral change in young adults with high-functioning autism receiving virtual reality social cognition training: a pilot study. Autism Res. 2018;11(5):713-25. doi: 10.1002/aur.1941.

10. Ip HH, Wong SW, Chan DF, Byrne J, Li C, Yuan VS, et al. Enhance emotional and social adaptation skills for children with autism spectrum disorder: a virtual reality enabled approach. Comput Educ. 2018;117:1-15. doi: 10.1016/j. compedu.2017.09.010.

11. Maskey M, Rodgers J, Grahame V, Glod M, Honey E, Kinnear J, et al. A randomised controlled feasibility trial of immersive virtual reality treatment with cognitive behaviour therapy for specific phobias in young people with autism spectrum disorder. J Autism Dev Disord. 2019;49(5):1912-27. doi: 10.1007/s10803-018-3861-x.

12. Newbutt N, Sung C, Kuo HJ, Leahy MJ, Lin CC, Tong B. Brief report: a pilot study of the use of a virtual reality headset in autism populations. J Autism Dev Disord. 2016;46(9):3166-76. doi: 10.1007/s10803-016-2830-5.

13. Welterlin A, Turner-Brown LM, Harris S, Mesibov G, Delmolino L. The home TEACCHing program for toddlers with autism. J Autism Dev Disord. 2012;42(9):1827-35. doi: 10.1007/s10803-011-1419-2.

14. Petralia MC, Sagone E, Coco M, Gueli R, Di Gregorio G, Coco LSG, et al. The 2D:4D ratio is associated with performance in the 'TEACCH program' of subjects with autism spectrum disorder. Exp Ther Med. 2018;16(6):53735. doi: 10.3892/etm.2018.6834.

15. Oliveira MA, Guebert M, Nohama P. TEACCH methodology-based web system to support learning for children with autism. IEEE Lat Am Trans. 2018;16(11):2698705. doi: 10.1109/tla.2018.8795110.

16. Moon SJ, Hwang JS, Shin AL, Kim JY, Bae SM, SheehyKnight J, et al. Accuracy of the Childhood Autism Rating Scale: a systematic review and meta-analysis. Dev Med Child Neurol. 2019;61(9):1030-8. doi: 10.1111/dmcn.14246.

17. Farmer C, Adedipe D, Bal V, Chlebowski C, Thurm A. Reliability of the Vineland Adaptive Behavior Scales. PsyArXiv; 2019. doi: 10.31234/osf.io/pn463.

18. Aman MG, McDougle CJ, Scahill L, Handen B, Arnold LE, Johnson C, et al. Medication and parent training in children with pervasive developmental disorders and serious behavior problems: results from a randomized clinical trial. J Am Acad Child Adolesc Psychiatry. 2009;48(12):1143-54. doi: 10.1097/CHI.0b013e3181bfd669.

19. McDougle CJ, Holmes JP, Bronson MR, Anderson GM, Volkmar FR, Price LH, et al. Risperidone treatment of children and adolescents with pervasive developmental disorders: a prospective open-label study. J Am Acad Child Adolesc Psychiatry. 1997;36(5):685-93. doi: 10.1097/00004583-199705000-00020.

20. Fung LK, Mahajan R, Nozzolillo A, Bernal P, Krasner A, Jo B, et al. Pharmacologic treatment of severe irritability and problem behaviors in autism: a systematic review and meta-analysis. Pediatrics. 2016;137 Suppl 2:S124-35. doi: 10.1542/peds.2015-2851K.

21. McCracken JT, McGough J, Shah B, Cronin P, Hong D, Aman $\mathrm{MG}$, et al. Risperidone in children with autism and serious behavioral problems. N Engl J Med. 2002;347(5):314-21. doi: 10.1056/NEJMoa013171.

22. Canitano R, Scandurra V. Risperidone in the treatment of behavioral disorders associated with autism in children and adolescents. Neuropsychiatr Dis Treat. 2008;4(4):723-30. doi: 10.2147/ndt.s1450.

23. Scahill L, Jeon S, Boorin SJ, McDougle CJ, Aman MG, Dziura J, et al. Weight gain and metabolic consequences of risperidone in young children with autism spectrum disorder. J Am Acad Child Adolesc Psychiatry. 2016;55(5):415-23. doi: 10.1016/j.jaac.2016.02.016.

24. Didehbani N, Allen T, Kandalaft M, Krawczyk D, Chapman S. Virtual reality social cognition training for children with high functioning autism. Comput Human Behav. 2016;62:703-11. doi: 10.1016/j.chb.2016.04.033.

25. Bellini S, Peters JK, Benner L, Hopf A. A meta-analysis of school-based social skills interventions for children with autism spectrum disorders. Remedial Spec Educ. 2007;28(3):153-62. doi: 10.1177/07419325070280030401. 